\title{
Time Variations in Elemental Abundances in Solar Energetic Particle Events
}

\author{
T. T. von Rosenvinge*, C. M. S. Cohen ${ }^{\dagger}$, E. R. Christian*, A. C. Cummings ${ }^{\dagger}$, R. A. \\ Leske $^{\dagger}$, R. A. Mewaldt ${ }^{\dagger}$, P. L. Slocum**, O. C. St. Cyr*, E. C. Stone ${ }^{\dagger}$ and M. E. \\ Wiedenbeck** \\ ${ }^{*}$ NASA/Goddard Space Flight Center, Greenbelt, MD 20771 USA \\ ${ }^{\dagger}$ California Institute of Technology, Pasadena, CA 91125 USA \\ ${ }^{* *}$ Jet Propulsion Laboratory, Pasadena, CA 91109 USA
}

\begin{abstract}
The Solar Isotope Spectrometer (SIS) on-board the Advanced Composition Explorer has a large collection power and high telemetry rate, making it possible to study elemental abundances in large solar energetic particle (SEP) events as a function of time. Results have now been obtained for more than 25 such events. Understanding the causes of these variations is key to obtaining reliable solar elemental abundances and to understanding solar acceleration processes. Such variations have been previously attributed to two models: (1) a mixture of an initial impulsive phase having enhanced heavy element abundances with a longer gradual phase with coronal abundances and (2) rigidity dependent escape from CME-driven shocks through plasma waves generated by wave-particle interactions. In this second model the injected abundances are assumed to be coronal. Both these models can be expected to depend upon solar longitude since impulsive events are associated with flares at longitudes well-connected magnetically to the observer, and shock properties and connection of the observer to the shock are also longitude dependent. We present results on temporal variations from event to event and within events and show that they appear to have a longitude dependence. We show that the events which have been well-explained by model (2) tend to be near central meridian or the west limb. In addition, we show that there are events with little time variation and heavy element enhancements similar to those of impulsive events. These events seem to be better explained by model (1) with only an impulsive phase.
\end{abstract}

\section{INTRODUCTION}

One of the goals of measurements of solar energetic particles (SEPs) has been to measure solar abundances of elements which are not easily determined by other methods. Cohen, et al. (1, this conference) present such results from the Solar Isotope Spectrometer (SIS)(2) onboard the Advanced Composition Explorer (ACE), averaged over the principal SEP events observed since the launch of ACE in August, 1997. The present paper illustrates the variations from event to event and within events that are integrated over by such averaging. These variations are substantial and illustrate the difficulties of this approach to obtaining solar abundances. Cohen, et al. (3), von Rosenvinge, et al. (4), and Boberg and Tylka (5) have also discussed variations in abundances measured by SIS, but for smaller numbers of events.

Event-to-event variations in elemental abundances have been known for a long time (e.g., 6,7,8,9; see also the review by Reames (10)). The causes of these varia- tions, however, have been relatively slow in being understood. An important step was the work of Breneman and Stone (8), who showed that the elemental abundances in specific solar events could be ordered as power laws in $Q_{X} / M_{X}$, where $Q_{X}$ and $M_{X}$ are the mean ionic charge state and the mean elemental mass for element $X$. The corresponding power laws had exponents ranging from roughly -2.5 to +2.5 , corresponding to both heavy element enhancements and heavy element depletions. No explanation for this behavior was provided other than to presume that particle rigidity was the essential parameter governing both particle acceleration and escape from the sun. While this approach was purely phenomenological, it did provide a means for estimating $Q / M$ dependent biases between the observed abundances and solar abundances.

Reames, et al. (11) and Cane, et al. (12) were the first to report on an organization of abundance data by the longitude of the source region. They suggested that solar event elemental abundances as a function of lon-

CP598, Solar and Galactic Composition, edited by R. F. Wimmer-Schweingruber (C) 2001 American Institute of Physics 0-7354-0042-3/01/\$18.00 
gitude could be understood in terms of two particle sources: 1) particles originating out of flare-heated material and 2) particles accelerated at coronal and interplanetary shocks. Fe-rich, high Q-state, flare-heated material would only have access to the observer when the observer was magnetically well-connected to the flare site. Reames (10) has subsequently rejected this interpretation, instead attributing the longitude dependence of Fe enhancements to the fact that the observer is wellconnected magnetically to the nose of a shock (where the shock is presumably the strongest) when the shock originates at a western location. The longitude dependence of SEP abundances has been largely ignored since the time of Cane, et al. (12) but will be revisited in the present paper. We note that the Cane, et al. (12) explanation is consistent with the correlation between $\mathrm{Fe} / \mathrm{O}$ and $\left\langle Q_{\mathrm{Fe}}\right\rangle$ reported at low energies by Möbius, et al. (13) and at high energies by Leske, et al. (14, this conference).

Recently instruments have been built which have much greater collection power than was available to Cane, et al. (12) and which can therefore examine time variations within many more events and in greater detail $(2,15)$. In principle this gives greater opportunities for understanding the causes of variations. Recent work by Reames, Tylka and $\mathrm{Ng}$ has explained some unexpected temporal behavior in solar event composition in terms of shock acceleration and the build-up of plasma waves, predominantly due to proton wave-particle interactions (e.g., 16,17,18; see also Reames (19), this conference). The theory of $\mathrm{Ng}$, et al. (18), however, is incomplete in that it posits a spectrum which is continuously injected at a moving shock and accounts for how particles are released from the shock and travel to the observer. This release depends upon the temporal build-up of plasma waves in the vicinity of the shock. In other words, the $\mathrm{Ng}$, et al. (18) model does not follow the acceleration of individual particles from low to high energies. Thus, for example, it does not readily explain why events such as the 20 April 1998 event are Fe-rich at the energies $(\sim 3-10 \mathrm{MeV} /$ nucleon) measured by the Low Energy Matrix Telescopes (LEMT) on-board the Wind spacecraft (16) and Fe-poor at SIS energies $(\sim 12-60$ $\mathrm{MeV} /$ nucleon) (4). Nonetheless, a qualitative explanation can be provided in the framework of the $\mathrm{Ng}$, et al. (18) model as discussed by von Rosenvinge, et al. (4).

The $\mathrm{Ng}$, et al. (18) model assumes that particles are injected into the shock with coronal abundances. This model can, however, create an enhancement in $\mathrm{Fe} / \mathrm{O}$ above the coronal value at low energies for some observers for at least a portion of the event. In particular, it has been able to inject particles with coronal abundances and produce a peak in the modeled $\mathrm{Fe} / \mathrm{O}$ time intensitycurve at a few MeV/nucleon as reported by Tylka, et al. (16) in the 1998 April 20 event. This peak was enhanced by a factor of 4 over the coronal value. A constant high value of $\mathrm{Fe} / \mathrm{O}$, however, would be difficult to achieve in this model.

The model of Ellison and Ramaty (20) produces a $Q / M$ dependent energy spectrum by accounting for first order Fermi acceleration and rigidity dependent escape at a shock, but the predicted spectral shape, unlike the model of $\mathrm{Ng}$, et al. (18), applies at the shock and not to the remote observer. Furthermore there is no time dependence in the Ellison and Ramaty (20) model. This model has fit differential intensity spectra quite well and has been useful in deriving $Q / M$ ratios for different elements (21). This is possible since the model spectrum consists of a power law in momentum modified by an exponential factor, $\exp \left(-E / E_{0 X}\right)$, produced by the finite spatial extent of the shock. Here $E$ is kinetic energy/nucleon and $E_{0 X}=E_{0 H}\left(Q_{X} / M_{X}\right)$, where $X$ denotes element $\mathrm{X}$ and $E_{0 H}$ is the appropriate $E_{0 X}$ for hydrogen. The exponential factor gives rise to characteristic spectral knees which can, for example, account for depletions of Fe relative to $\mathrm{O}$ above $\sim E_{0 F e}$ since in general $Q_{F e} / M_{F e}$ is less than $Q_{O} / M_{O}$. Tylka, et al. (21), by dividing event data into time intervals of 8 hours, have used the evolution of spectral shapes to infer charge states as a function of time during two events. However, it is hard to see how this model could account for the $\mathrm{Fe} / \mathrm{O}$ ratio increasing with energy as was observed for the 6 November 1997 event $(16,4)$. It is also not clear how it could account for the previously mentioned correlation between $\mathrm{Fe} / \mathrm{O}$ and $\left\langle Q_{F e}>\right.$ or for enhancements above coronal abundances.

\section{OBSERVATIONS}

The Solar Isotope Spectrometer (SIS) on-board the Advanced Composition Explorer (ACE) has a large geometry factor $\left(\sim \mathrm{cm}^{2}\right.$-sr), enabling abundances in solar energetic particle (SEP) events to be observed on a time scale of hours or less. We have identified 27 such events, which are listed in Table 1 . The columns labeled 'Start UT' and 'End UT' give the start and end Universal Times of the time interval for which energetic particle intensities were studied for each event. Table 1 includes approximate times and solar longitudes for 'associated' solar flares. The identification of such flares is not always straight forward. Indeed, in models where acceleration in gradual events is due to acceleration by shocks driven by Coronal Mass Ejections (CMEs), 'associated' solar flares are not even considered to be relevant. Nonetheless, we have attempted to find such flares and their start times and longitudes for each particle event using $H_{\alpha}, \mathrm{X}$-ray, and Type II and Type III radio data taken mainly from NOAA's Solar Geophysical Data Reports (http: / / julius.ngdc.noaa.gov: $8000 /$ Welcome_SGD.html). Radio data was also 


\begin{tabular}{|c|c|c|c|c|c|}
\hline$\#$ & Start UT & End UT & Flare UT & Longitude & Comments \\
\hline 1 & 1997 Nov 4 06:00 & 1997 Nov 6 12:00 & Nov 5 05:52 & W33 & $\mathrm{X} 2.1,2 \mathrm{~B}$ \\
\hline 2 & 1997 Nov 6 12:00 & 1997 Nov 10 00:00 & Nov 6 11:49 & W63 & X9.4, 2B \\
\hline 3 & 1998 Apr 20 12:00 & 1998 Apr 27 00:00 & Apr 20 09:38 & W90 & $\mathrm{M} 1.4$, no $\mathrm{H}_{\alpha}$ \\
\hline 4 & 1998 May 2 12:00 & 1998 May 5 00:00 & May 2 13:31 & W15 & $\mathrm{X} 1.1,3 \mathrm{~B}$ \\
\hline 5 & 1998 May 6 08:00 & 1998 May 8 00:00 & May $607: 58$ & W65 & $\mathrm{X} 2.7,1 \mathrm{~N}$ \\
\hline 6 & 1998 May $904: 50$ & 1998 May 11 12:00 & May 9 03:04 & W90 & M7.7, no $H_{\alpha}$ \\
\hline 7 & 1998 Aug 25 00:00 & 1998 Sep 1 00:00 & Aug 24 21:50 & E09 & $\mathrm{X} 1.0,3 \mathrm{~B}$ \\
\hline 8 & 1998 Sep 24 12:00 & 1998 Sep 25 12:00 & Sep 23 07:13 & E09 & $\begin{array}{l}\text { M7.1, 3B, strong ESP spike after Sep } 24 \\
\text { 12:00 UT }\end{array}$ \\
\hline 9 & 1998 Sep 30 12:00 & 1998 Oct 5 00:00 & Sep 30 13:50 & W78 & $\mathrm{M} 2.8,2 \mathrm{~N}$ \\
\hline 10 & 1998 Oct $1900: 00$ & 1998 Oct 20 18:00 & Oct $1821: 15$ & W90 & no X-rays, no $\mathrm{H}_{\alpha}$, Type III \\
\hline 11 & 1998 Nov 14 06:00 & 1998 Nov 18 12:00 & Nov 14 05:05 & W90 & weak X-rays, no $\mathrm{H}_{\alpha}$ \\
\hline 12 & 1999 Jan 21 00:00 & 1999 Jan 27 12:00 & Jan 20 19:06 & W90 & $\mathrm{M} 5.2$, no $\mathrm{H}_{\alpha}$ \\
\hline 13 & 1999 Feb 16 00:00 & 1999 Feb 19 00:00 & Feb 16 02:49 & W14 & $\mathrm{M} 3.2, \mathrm{SF}$ \\
\hline 14 & 1999 Apr 24 12:00 & 1999 Apr 26 12:00 & Apr 24 12:36 & W90 & no $\mathrm{X}$-rays, no $\mathrm{H}_{\alpha}, \mathrm{CME}$ \\
\hline 15 & 1999 May 4 00:00 & 1999 May 9 12:00 & May 3 05:50 & E32 & $\mathrm{M} 4.4,2 \mathrm{~N}$ \\
\hline 16 & 1999 May 9 18:00 & 1999 May 11 00:00 & May 9 18:07 & W90 & $\mathrm{M} 7.6$, no $\mathrm{H}_{\alpha}$ \\
\hline 17 & 1999 Jun 2 00:00 & 1999 Jun 4 08:00 & Jun 1 19:30 & W90 & no $\mathrm{X}$-rays, no $\mathrm{H}_{\alpha}, \mathrm{CME}$ \\
\hline 18 & 1999 Jun 4 08:00 & 1999 Jun 8 00:00 & Jun 4 07:00 & W69 & M3.9, 2B \\
\hline 19 & 2000 Apr 4 12:00 & 2000 Apr 9 00:00 & Apr 4 15:12 & W66 & $\mathrm{C} 9.7,2 \mathrm{~F}$ \\
\hline 20 & 2000 Jun 7 00:00 & 2000 Jun 10 00:00 & Jun 6 13:24 & E12 & $\mathrm{X} 1.1,3 \mathrm{~B}$ \\
\hline 21 & 2000 Jun 10 12:00 & 2000 Jun 13 00:00 & Jun 10 16:40 & W38 & M5.2, 3B \\
\hline 22 & $2000 \mathrm{Jul} 14$ 00:00 & 2000 Jul 19 00:00 & Jul 14 10:03 & W07 & X5.7, 3B \\
\hline 23 & 2000 Sep 12 12:00 & 2000 Sep $1600: 00$ & Sep 12 13:31 & W05 & \\
\hline 24 & 2000 Oct $1600: 00$ & 2000 Oct $2000: 00$ & Oct $1607: 00$ & W90 & $\mathrm{M} 2.5$, no $\mathrm{H}_{\alpha}$ \\
\hline 25 & 2000 Oct 25 12:00 & 2000 Oct 29 00:00 & Oct 25 09:30 & W90 & no $\mathrm{X}$-rays, no $\mathrm{H}_{\alpha}$ \\
\hline \multirow[t]{2}{*}{26} & 2000 Nov 9 00:00 & 2000 Nov 15 00:00 & Nov 8 23:28 & W77 & $\mathrm{M} 7.4,3 \mathrm{~F}$ \\
\hline & & & Nov 9 16:13 & E10 & M1.0, SF \\
\hline \multirow[t]{3}{*}{27} & 2000 Nov 24 00:00 & 2000 Nov 29 00:00 & Nov 24 05:02 & W05 & $\mathrm{X} 2.0$ \\
\hline & & & Nov 24 15:13 & W07 & $\mathrm{X} 2.3$ \\
\hline & & & Nov 24 21:59 & W14 & $\mathrm{X} 1.8$ \\
\hline
\end{tabular}

taken from the WAVES investigation on the Wind spacecraft (http://lep694.gsfc.nasa.gov/ waves (waves.html). CME data was provided from the LASCO instrument on the SoHO spacecraft. For the simplest cases, a hard X-ray event starts essentially at the same time as a Type III burst, followed by $H_{\alpha}$ and highfrequency Type II emission. Utilizing the Wind/WAVES radio data, the Type III data can be generally tracked down to $\sim 20-40 \mathrm{kHz}$, the local plasma frequency at 1 A.U., indicating magnetic connection between the particle acceleration site all the way out to the observer at 1 A.U. The event solar longitude is then obtained from the $H_{\alpha}$ data. More difficult cases have no $H_{\alpha}$ event, and even no X-ray event. These events are generally from beyond the west limb of the sun, as can be verified by, for example, LASCO CME observations. While one can perhaps obtain a better location by extrapolating observations of active regions prior to their rotating out of view, we have simply entered the location in Table 1 as $W 90$. In some cases there are multiple flare events and these are indicated in Table 1. Figures 1, 2 , and 3 show event-averaged abundances for different events as observed by SIS in the energy interval 12-60
$\mathrm{MeV} /$ nucleon. The energy interval which SIS covers for each element varies with the atomic number Z. 12-60 $\mathrm{MeV} /$ nucleon is the broadest energy interval for which SIS can observe all elements from $\mathrm{C}$ to Fe. Figures 1, 2 , and 3 show SIS abundances versus $\mathrm{Z}$ as ratios with respect to the average abundances for gradual events (open circles) and for impulsive events (closed circles) as reported by Reames (22). The average gradual event values are thought to represent average coronal values (22). Figures 1,2, and 3 show data for sample events which are, respectively, depleted, unchanged, and enhanced in heavy elements relative to the average abundances in gradual events. These figures show that heavy element enhancements/depletions vary considerably from event to event but that each event generally shows a trend with respect to increasing $\mathrm{Z}$.

The upper three panels of Figure 1 show large depletions of heavy elements corresponding to events east of central meridian and, in the rare case of the 20 April 1998 event, at $\sim W 90$. The bottom panel corresponds to 2 widely separated events, one at W77 and one at E10, as shown by the corresponding Type III bursts which both reach 1 A.U. Figure 2 shows three events near central 

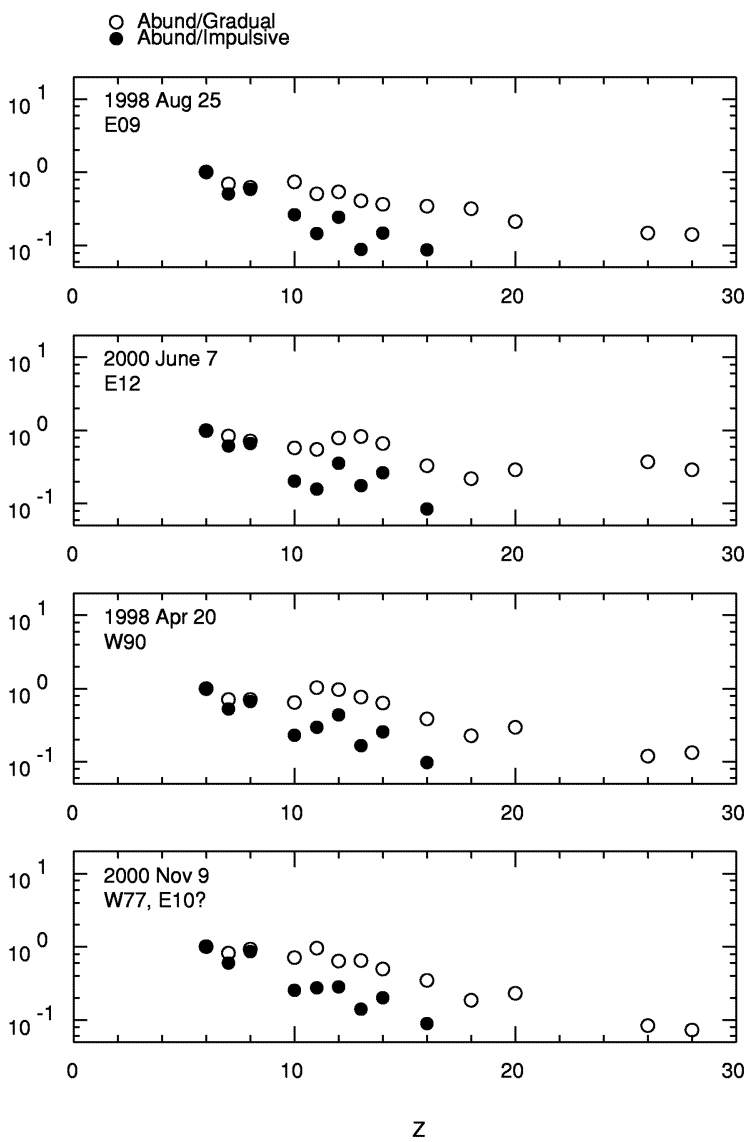

FIGURE 1. Shows the ratios of element abundances observed by SIS to those of average gradual events (open circles) and to those of average impulsive events (closed circles). Each panel shows these ratios normalized to $\mathrm{C}$ and plotted versus $\mathrm{Z}$ for a separate SIS particle event. These events were chosen because they show heavy-element depletions relative to average gradual event abundances.

meridian, one at W66 and one from beyond the west limb. Except for event 19 at W66 these events follow a similar pattern to the events of Figure 1. Figure 3 shows that events with impulsive-like composition can come from all longitudes $\gtrsim W 15$.

Figure 4 shows the enhancement of $\mathrm{Fe} / \mathrm{C}$ over the average gradual event $\mathrm{Fe} / \mathrm{C}$ ratio for multiple events versus the solar longitude of the associated flare event (i.e. the $y$-axis values are the same as for the open circles at $\mathrm{Z}=26$ in Figures 1, 2, and 3). The ACE-SIS event number from Table 1 is printed for each event in place of a data point. This figure shows that all events occurring between $W 15$ and $W 85$ have $\mathrm{Fe}$ enhancements greater than one. All events with enhancements $\sim 1$ or less are either to the east of $W 15$ or, in the case of the unusual 20 April 1998 event, at $\sim W 90$. Events with large Fe enhancements also occur at or beyond the west limb, but not near central meridian.
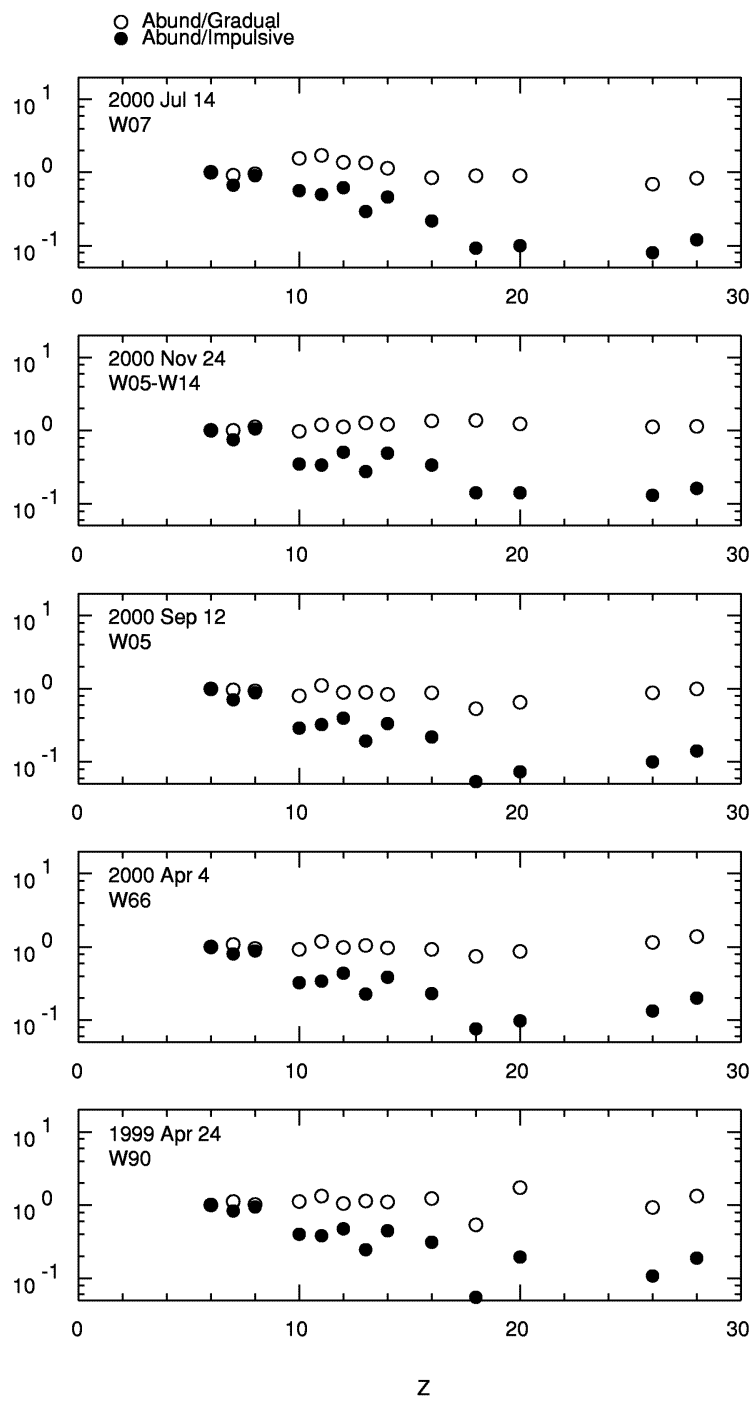

FIGURE 2. Similar to Figure 1, but for particle events with abundances similar to average gradual event abundances.

Figure 5 shows, for each separate event, the ratio of the maximum $\mathrm{Fe} / \mathrm{O}$ ratio to the minimum $\mathrm{Fe} / \mathrm{O}$ ratio versus the solar longitude of the associated flare event. The ratios are determined at $14 \mathrm{MeV} /$ nucleon. This then gives a measure of the variability of $\mathrm{Fe} / \mathrm{O}$ during each event. We see that, with the exception of event 19 , there is very little variability within events between $\sim W 15$ and $\sim W 80$. Events with relatively large variability are either close to central meridian or towards the west limb. Events with low variability, however, can occur at all longitudes.

Figure 6 shows time-intensity profiles (upper panel) and ratios (lower panel) at $14 \mathrm{MeV} /$ nucleon normalized to average gradual event values for representative elements in the event of 2 May 1998. This normalization 

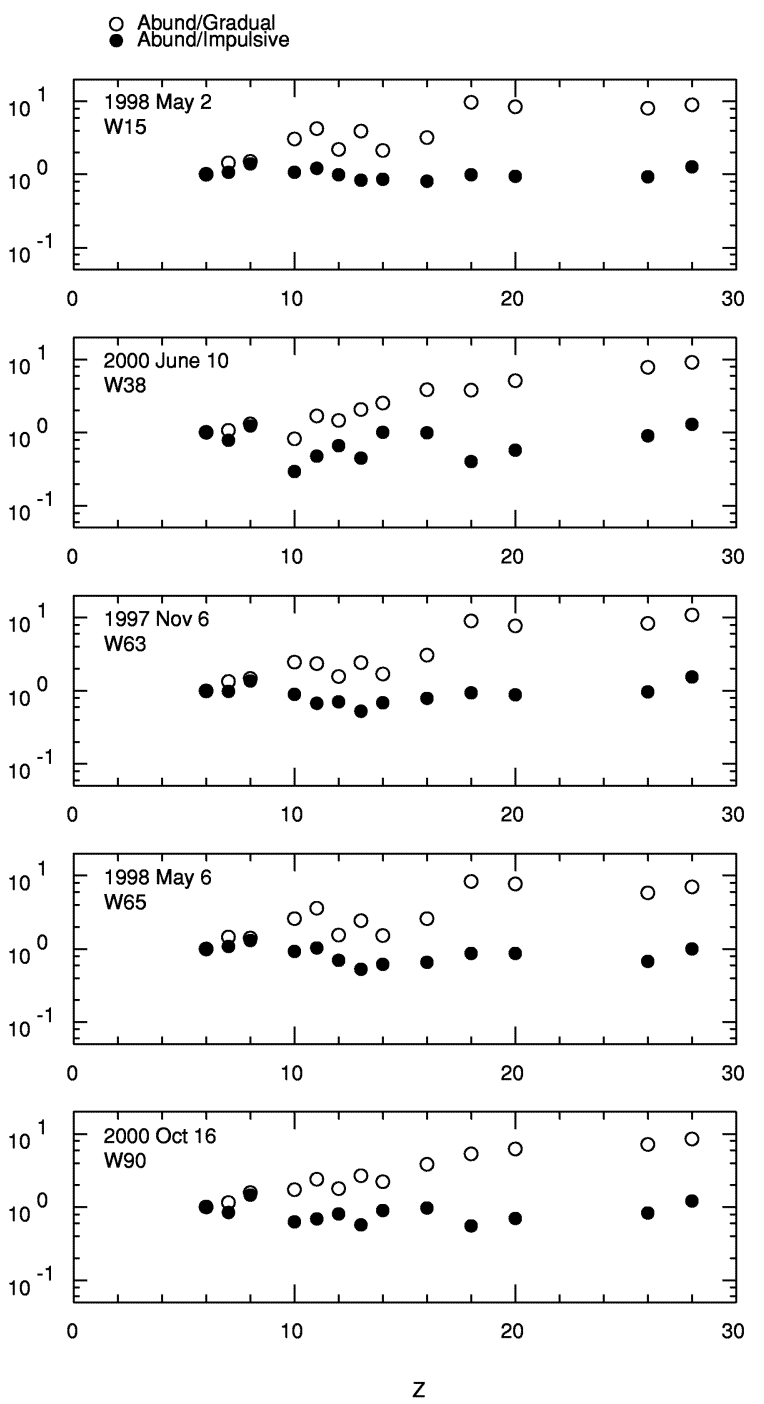

FIGURE 3. Similar to Figure 1, but for particle events with abundances similar to average impulsive event abundances.

means that ratios which are the same as the corresponding event averages given by Reames (22) are plotted with a value of 1.000 and enhancements above (or depletions below) the average values are immediately apparent. There was a corresponding flare at $W 15$ at 1998 May 2 13:31 UT. Figure 6 shows that the normalized Fe/O ratio is quite constant throughout the event with a value of approximately 6 . It also shows that the normalized $\mathrm{Ca} / \mathrm{O}$ ratio is similarly constant with a similar value. The ratios $\mathrm{He} / \mathrm{O}$ and $\mathrm{Si} / \mathrm{O}$ show only slight enhancements. Note also the two interplanetary shocks shown in Figure 6 and separated by only 9 hours. The presence of these two shocks potentially complicates the interpretation of this event. On the other hand, as shown in Figures 4 and 5, wellconnected events with relatively constant $\mathrm{Fe} / \mathrm{O}$ during

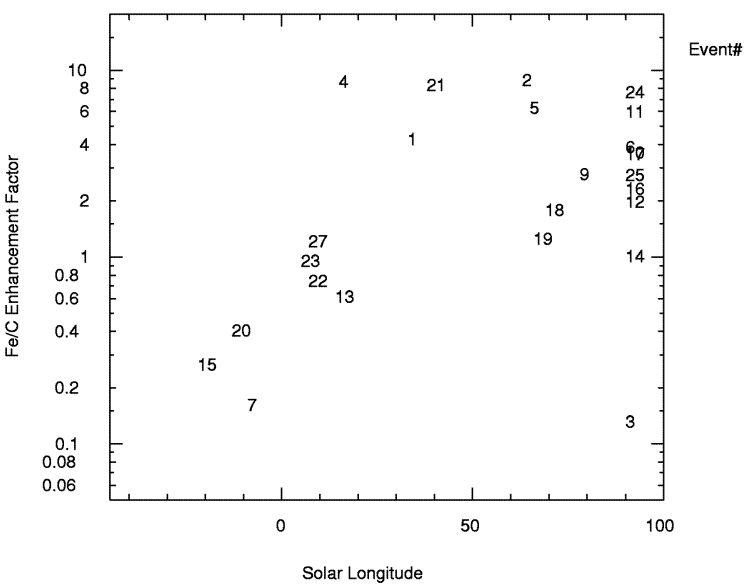

FIGURE 4. Shows the enhancement of $\mathrm{Fe} / \mathrm{C}$ over the average gradual event abundance reported by Reames (1995) for multiple events versus the solar longitude of the associated flare event.

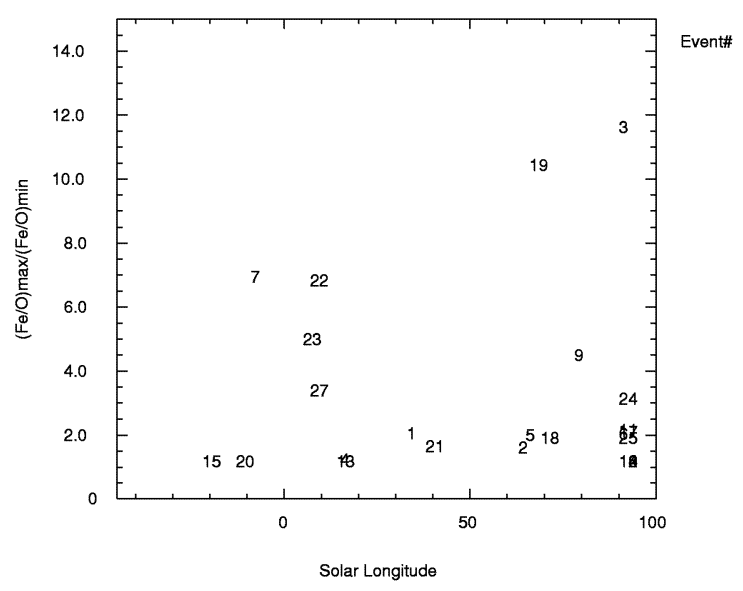

FIGURE 5. Shows the ratio of the maximum $\mathrm{Fe} / \mathrm{O}$ ratio during each event to the minimum $\mathrm{Fe} / \mathrm{O}$ ratio versus the solar longitude of the associated flare event.

each event and large enhancements of $\mathrm{Fe}$ are fairly common. Three other events with relatively constant Fe/O (6 November 1997, W33, 14 November 1998, > W90, and 6 May 1998, W65) are shown in von Rosenvinge, et al. (4) and von Rosenvinge, et al. (23).

\section{DISCUSSION}

It is clear from the data presented here that the observed event-to-event average abundances depend on the solar longitude of associated solar flare events. It appears that the temporal variability of abundances within individual events may also depend upon solar longitude. At this point there is no accepted model which we know of that 

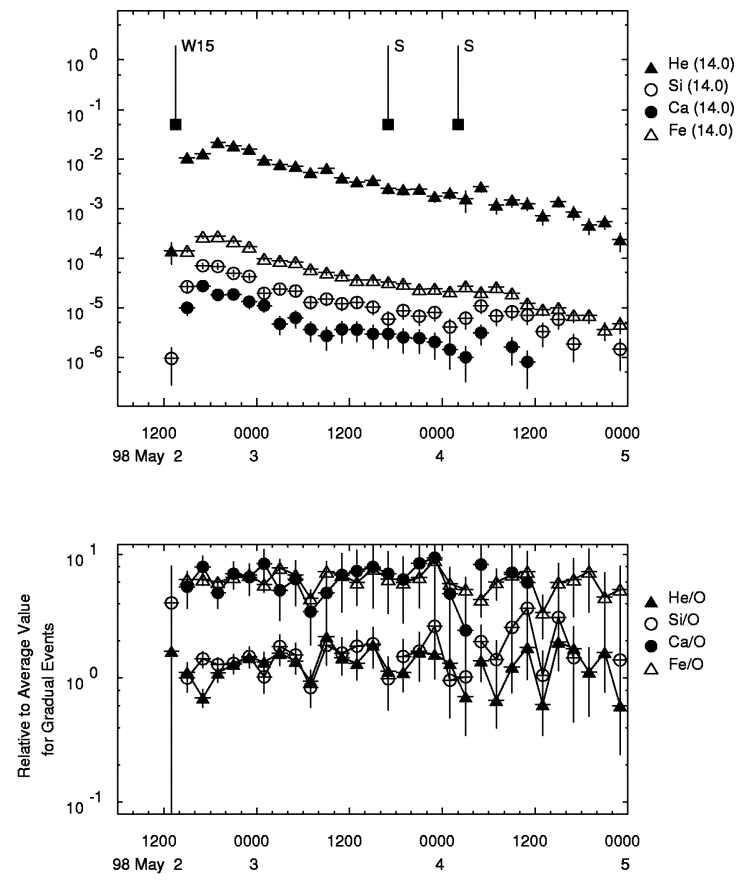

FIGURE 6. Example of an event where $\mathrm{Fe} / \mathrm{O}$ is essentially constant throughout the event at an enhanced level.

accounts for these dependences, although the suggestion of Cane, et al. (21) that there can be a major flare accelerated component cannot be ruled out. In fact, this suggestion is consistent with the observation that the mean $\mathrm{Fe}$ charge state in large events is correlated with $\mathrm{Fe} / \mathrm{O}$ (13, 14).

We have also shown that events originating from beyond $\sim W 15$ commonly show large $\mathrm{Fe} / \mathrm{O}$ enhancements which vary little during the course of the events. Such enhancements cannot be explained within the context of the Ellison and Ramaty (20) model and injection of relative abundances at low energies corresponding to coronal abundances. It is possible that injections from multiple impulsive events can fill the inner solar system with enhanced heavy elements which are then accelerated by CME related shocks (Mason, et al., 24). However, events with large $\mathrm{Fe} / \mathrm{O}$ enhancements have heavy element composition essentially identical to that of impulsive events, so this process would have to completely dominate the acceleration of normal coronal/solar wind material. And why, then, would there be no Fe-enhanced events east of $\sim W 15$ ?

It is not clear either how the $\mathrm{Ng}$, et al. (18) model can account for events with large, constant $\mathrm{Fe} / \mathrm{O}$ enhancements. The Ellison and Ramaty (20) model, however, does appear to have successfully accounted for spectral shapes for elements from $\mathrm{H}$ to $\mathrm{Fe}$ for the event of 20 April 1998 (at the west limb) and the event of 25 August 1998 (at E09) (Tylka, et al., 21). Similar analysis of spectral shapes for events with near constant Fe enhancements has yet to be done. It similarly appears that the model of $\mathrm{Ng}$, et al. (18) applies very well to events for which the observer is poorly connected to the associated flare region, most notably events east of $\sim W 15$. Many events west of $W 15$, however, may require a flare-related component.

\section{ACKNOWLEDGMENTS}

This research was supported by the National Aeronautics and Space Administration at the Goddard Space Flight Center, the California Institute of Technology (under grant NAG5-6912), and the Jet Propulsion Laboratory. TvR acknowledges assistance from Hilary Cane with determining associated solar event locations.

\section{REFERENCES}

1. Cohen, C.M.S., et al., ACE/SoHO Workshop Proc., Berne, this volume (2001).

2. Stone, E.C., et al., Sp. Sci. Rev., 86, 357 (1998).

3. Cohen, C.M.S., et al., Geophys. Res. Lett., 26, 2697 (1999).

4. von Rosenvinge, et al., Proc. 26th Int. Cosmic Ray Conf. (Salt Lake City), 6, 131 (1999).

5. Boberg, P.R. and Tylka, A.J., AIP Conf. Proc., 528, 115 (2000).

6. Teegarden, B.J., et al., Ap. J., 180, 571 (1973).

7. McGuire, et al., Ap. J., 301, 938-961 (1986).

8. Breneman, H.H. and Stone, E.C., Ap. J. Lett., 299, L57-L61 (1985).

9. Reames, et al., Ap. J. Supp., 90, 649-667 (1994).

10. Reames, D.V., Sp. Sci. Rev., 90, 413 (1999).

11. Reames, D.V., et al., Ap. J., 357, 259 (1990).

12. Cane, H.V., et al., Ap. J., 373, 675 (1991).

13. Möbius, et al., AIP Conf. Proc., 528, 131-134 (2000).

14. Leske, R.A., et al., ACE/SoHO Workshop Proc., Berne, this volume, 2001.

15. von Rosenvinge, T.T., et al., Sp. Sci. Rev., 71, 155-206 (1995).

16. Tylka, A.J., et al., Geophys. Res. Lett., 26, 3585 (1999).

17. Reames, D.V., et al., Ap. J. Lett., 531, L83 (2000).

18. Ng, C.K., et al., Geophys. Res. Lett., 26, 2145 (1999).

19. Reames, D.V., ACE/SoHO Workshop Proc., Berne, this volume, 2001.

20. Ellison, D., and Ramaty, R., Ap. J., 298, 400-408 (1985).

21. Tylka, A.J., et al., AIP Conf. Proc., 528, 147 (2000).

22. Reames, D.V., Adv. Space Res., 15, 41 (1995).

23. von Rosenvinge, T.T., et al., AIP Conf. Proc., 528, 111 (2000).

24. Mason, G.M., et al., Ap. J. Lett., 525, L133 (1999). 